\title{
RAM 发电机无法正常建压的故障分析
}

\section{Fault analysis of Ram generator unable to build voltage normally}

\author{
俞仰峰 朱正林 王泽健 \\ Yangfeng Yu Zhneglin Zhu Zejian Wang \\ 中广核核电运营有限公司 中国·广东深圳 518000
}

China Nuclear Power Operations Co., Ltd. Shen Zhen, Guangdong, 518000, China

\begin{abstract}
摘要：某核电厂现场更换 RAM 发电机后，在现场进行空载试验时，发现发电机励磁无法正常建压，导致发电机无 法输出额定电压。本文详细介绍了 RAM 发电机励磁调节原理及故障处理过程。该分析可供同型号 RAM 发电机故障分 析提供参考。

Abstract: After replacing THEM generator on site, when carrying out an empty test on site, it was found that the generator excitation could not be properly constructed, resulting in the generator not being able to output the rated voltage. This paper introduces in detail the principle of excitation regulation of RAM generator and the fault treatment process. This analysis can be used as a reference for fault analysis of the same type of RAM generator.
\end{abstract}

关键词：发电机；励磁；电压

Keywords: Generator; Excitation; Voltage

DOI: $10.36012 /$ etr.v2i9.2672

\section{1 问题描述}

某核电厂 2 号机组第一次大修，电气人员持票进行 RAM 发电机年检工作, 发现发电机转子对地绝缘低, 更换 发电机后, 在现场进行空载试验时, 发现发电机励磁无法正 常建压, 导致发电机无法输出额定电压。

\section{RAM 系统简介}

RAM 系统为控制棒驱动机构电源系统, 其任务就是为 控制棒驱动机构供电, 由两台带惯性飞轮的电动机一发电机 组、励磁调节机构、电动机供电线路, 发电机输出线路以及 相关的电气保护线路等组成。RAM 系统包含两套装置, 正 常运行时, 二套装置并列运行, 经整流后, 向反应堆控制棒 提供驱动电源，各承担负荷的 $50 \%$ 。

每套装置的组成为分别由一台 $380 \mathrm{~V} 、 135 \mathrm{KW}$ 、转速 $1480 \mathrm{r} / \mathrm{min}$ 的三相异步电动机来带动一台 $400 \mathrm{KVA}$ 的发电机
和中间飞轮而转动, 并发出 $260 \mathrm{~V} / 50 \mathrm{HZ}$ 的三相交流电, 利 用该飞轮的惯性转动, 可以保证发电机在满负荷功况下, 失 去加在电动机上的动力电源 1.2 秒的时间内, 保证发电机输 出的电压在 $234 \mathrm{~V}$ 以上，频率保持在 $44 \mathrm{~Hz}$ 以上。

\section{RAM 发电机励磁调节原理}

根据发电机的外特性曲线，如下图所示。当发电机 励磁电流一定时, 发电机端电压随发电机定子电流变化的 曲线。

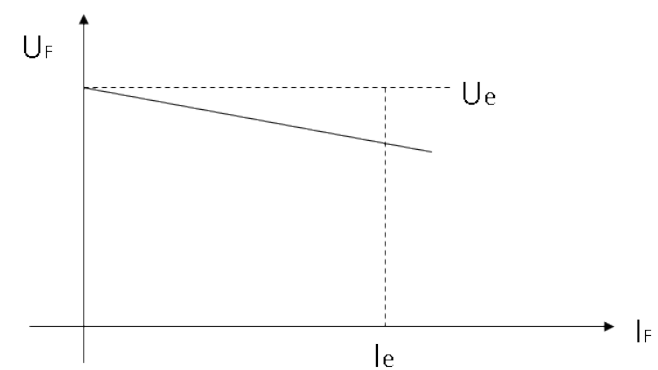

【作者简介】俞仰峰（1990 ）, 男, 福建福州人, 工程师, 学士学位, 从事低压交直流电动机及发电机研究。 
从外特性曲线可以看出, 随着负荷电流 I 的增加, 发电 机电枢反应去磁作用增强, 使发电机端电压越来越降低。为 了使 RAM 发电机端电压稳定, 即随着发电机负荷电流 I 的 增加而相应地增加发电机的励磁电流, 从而补偿发电机电枢
反应的去磁作用, 于是 RAM 采用了复式励磁装置, 在复式 励磁装置作用下, 励磁电流大小与发电机负荷电流成正比, 即可基本维持发电机端电压不变。

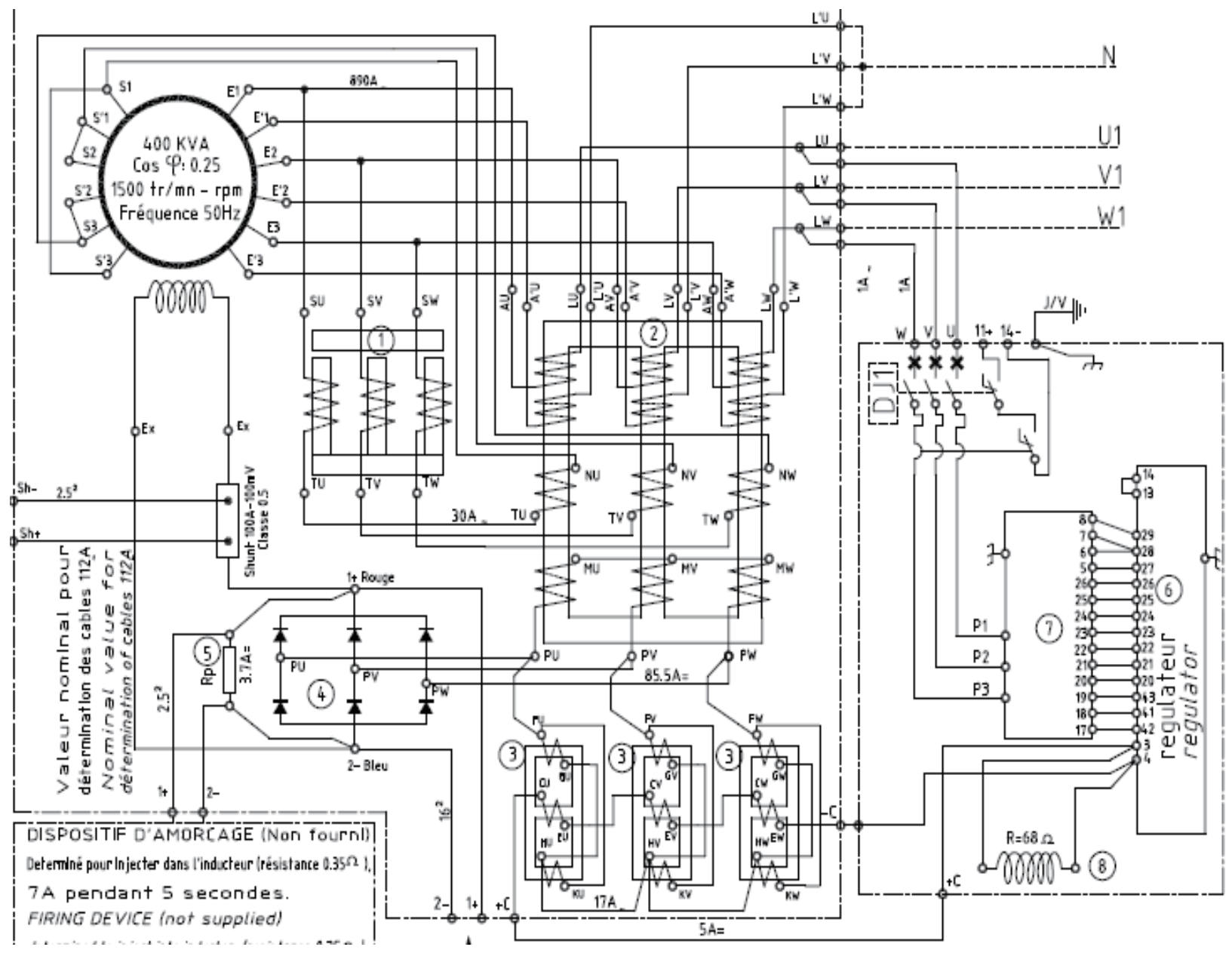

1 ）当发电机启动时：

发电机启动时, 由外加直流电源直接接入 1+、2- 号线, 提供励磁电流，5秒后断开，改由 RAM 发电机自励系统提 供励磁。(2)三相变压器将发电机输出的定子电流转换成励磁 电流, 从 PU/PV/PW 抽头引出, 再经过(4)三相整流桥整流后, 给发电机励磁供电，提供自励。

2 ) 发电机带载运行时:

当负荷电流较大, (2)三相变压器输出的电流也大, 而 使发电机励磁电流也大。当负荷电流减小时, (2)三相变压器 输出电流也小, 而使发电机励磁电流也减小。当发电机机端 或母线上故障 (短路) 时, 定子电流很大, 三相变压器(2)二
次电流也会很大, 使励磁电流急速增加, 发电机强励, 保护 动作可实现发电机自动停运。

(2)三相变压器铁芯的磁通量是由 $\mathrm{T} / \mathrm{N}$ 绕组和发电机定 子绕组中的电流决定的, 由右手定则可以判断, 在每一相中 二者电流产生的磁通方向相同, 所以(2)三相变压器铁芯中的 的总磁通量由定子绕组与 $\mathrm{T} / \mathrm{N}$ 绕组两者产生磁通量的叠加。 如果 $\mathrm{T} / \mathrm{N}$ 绕组中流过的电流增大, 则变压器中的总磁通量 增大, 相应的, $\mathrm{PU} / \mathrm{PV} / \mathrm{PW}$ 抽头输出并经整流器整流后的励 磁电流也增大。

\section{(2)三相变压器的 $\mathrm{T} / \mathrm{N}$ 绕组与 (1)三相可调阻抗 $\mathrm{S} / \mathrm{T}$ 绕组} 串联, 并从定子绕组 $\mathrm{E} / \mathrm{S}$ 绕组取电。以 $\mathrm{U}$ 相为例, $\mathrm{TU}-\mathrm{NU}$ 
与 TU-SU 串联后接入定子绕组 E1-S1, 如下图所示。

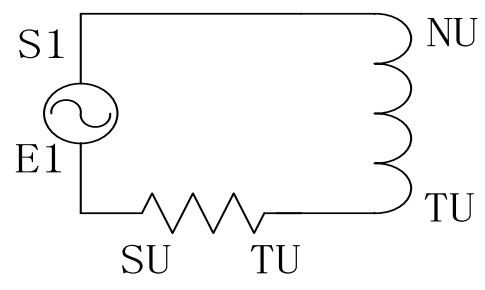

当发电机稳定运行时，定子绕组 E1-S1 的输出电压基 本保持不变, 回路电流大小由 TU-NU 与 TU-SU 绕组的阻 抗大小决定, 阻抗是由电阻与感抗组成的, 其中 TU-NU 与 TU-SU 的电阻值极小, 与感抗值相比可忽略不计, 且 TU-NU 绕组的感抗值不变, 故该回路电流大小主要是由 TU-SU 绕组的感抗值决定的。

$$
\mathrm{R}_{\text {感抗 }}=\omega \mathrm{L}=2 \pi \mathrm{f} * \mu \frac{N^{2} S}{l}
$$

对于(1)三相可调线圈的 TU-SU 绕组, 增加空气间隙使 磁导率 $\mu$ 减小, 磁通路径 $l$ 增加, 沛数 $\mathrm{N}$ 和磁通截面 $\mathrm{S}$ 不 变, 故而 $\mathrm{L}$ 减小, $\omega \mathrm{L}$ 减小, 回路电流增大, $\mathrm{TU}-\mathrm{NU}$ 绕组 中流过的电流增大, 则变压器铁芯中的总磁通量增大, 从而 $\mathrm{PU}$ 抽头输出的励磁电流也增大, 其他两相 ( $\mathrm{V}$ 相 $\mathrm{W}$ 相) 的 分析同理。

外接的励磁调节器通过监测发电机输出电压, 输出一 个直流电源来控制(3)单相饱和线圈的磁阻大小，从而控制励
磁电流输出的大小, 达到控制和调压的目的。具体控制逻辑 如下: 当监测到的发电机负荷电流增大，使发电机端电压下 将时, 励磁调节器输出的电流减小, 励磁调节器输出的电流 送至(3)单相饱和线圈，使(3)单相饱和线圈的等效磁导率增 大，从而感抗 XL 增大，从 $\mathrm{PU} / \mathrm{PV} / \mathrm{PW}$ 抽头分流的电流就相 应减小，由于负荷电流的增大，(3)单相饱和线圈的分流电流 减小, 流入 (4)三相整流桥的电流增大, 对应励磁电流就增大, 最终使发电机输出电压增大, 实现调节。发电机端电压上升 时的调节过程与之相反。

\section{4 故障原因分析}

现场检查发现新发电机上三相可调线圈铁芯铁颚处无 绝缘板, 而旧发电机有绝缘板，绝缘板的厚度即影响上述分 析中的三相可调线圈空气间隙的大小，垫上绝缘板后即相当 于增加了铁芯与绕组间的空气间隙，使阻抗降低，流过其的 电流增加, 经过三相变压器耦合使励磁电流增加, 从而提高 发电机出口电压。

\section{5 结论}

本次 RAM 发电机无法正常建压的故障,通过发现故障, 分析故障，解决故障。为今后检修同类设备积累了宝贵的 经验，同时也保证了 RAM 发电机的安全稳定运行。对后 续有刷发电机同类问题的分析处理具有很高的参考和借鉴 价值。 